\title{
Difusión de humedad en mezclas de moldeo para machos de fundición
}

\author{
J. E. HERNÁNDEZ-RUIZ, E. VALENCIA- MORALES, E. VILLAR-COCIÑA Y J. VEGA- LEIVA \\ Departamento de Física. Universidad Central de Las Villas. Santa Clara.54 830. V.C. Cuba.
}

Se describe la difusión de humedad en mezclas de moldeo utilizadas en los procesos de fundición. Empleando técnicas gravimétricas se registra el comportamiento de la cinética de humectación de diferentes tipos de mezclas de moldeo para machos de fundición. La higroscopicidad de las mezclas es comparada usando el criterio del incremento porcentual en masa húmeda. El estudio cinético se concluye con los cálculos de los coeficientes efectivos de difusión del agua atmosférica en las mezclas a distintas intemperies. Para este propósito se emplean métodos de ajuste no lineal. También se analiza la influencia de los materiales aglutinantes y desarenantes en las propiedades higroscópicas de estas mezclas.

Palabras claves: Humedad, difusión y mezclas de moldeo.

\section{Moisture diffusion in molding mixtures for cores of founding}

The moisture diffusion in molding mixtures employing in foundry processes is described. Using gravimetric techniques the humectation kinetic behavior of different types of molding mixtures for foundry cores were registered. The mixture higroscopicity is compared using the porcentual increase in wet mass.

The kinetic study is ending with the calculations effective coefficients of diffusion of the atmospheric water in the mixtures to different bleakness. For this goal the fitting through non lineal methods was used. The influences of the agglutinative and draw materials in the hygroscope properties of these were analyzed too.

Key words: Moisture, diffusion and molding mixtures.

\section{INTRODUCCIÓN}

Ya desde los años 70 son múltiples las ventajas que hacen retomar la práctica de los moldes de arena frente a las nuevas tecnologías que surgían en las fundiciones de hierro, dado entre otros factores por sus inferiores costos y ausencia de contaminación del medio ambiente ${ }^{(1,2)}$. Entre las limitaciones de esta práctica se encuentra el deterioro de mezclas para moldes y machos por concepto de su incremento en masa húmeda, como consecuencia de las prolongadas exposiciones a la intemperie ${ }^{(3)}$. Por ello, la mejora de la calidad en las producciones fundidas, que emplean moldes y machos de arena es un problema necesario y actual.

El incremento porcentual en masa húmeda de equilibrio para una mezcla dada a una determinada intemperie puede ser usado como medida del grado de hidratación de la mezcla ${ }^{(4)}$. Sin embargo, esto es una descripción incompleta del problema. La caracterización integral de la cinética de humectación del material incluye el cálculo de los coeficientes efectivos de difusión del agua ambiental en la mezcla de moldeo ${ }^{(3)}$.

La solución del problema difusivo depende de la geometría que se seleccione ${ }^{(3,5,6)}$. Por un problema de simplicidad se requiere la elección de las muestras experimentales con geometrías lo más sencillas y apropiadas para los cálculos.

Si una muestra tipo pastilla finita, permeable por una cara e inicialmente seca se expone a una intemperie con humedad constante ${ }^{(3)}$, se pueden presentar dos situaciones ${ }^{(3,5)}$ :

1. La difusión transcurre en un medio finito,

\section{LA DIFUSIÓN TRANSCURRE EN UN MEDIO SEMIINFINITO}

La solución del primer problema de contorno, resolver la ecuación de difusión para una pastilla finita y permeable por una cara (para el caso en que $D=c t e$ ) puede ser escrita en la forma ${ }^{(3,5,6)}$ :

$$
m_{\mathrm{t}} / m_{\infty}=1-\left(8 / \pi^{2}\right) \sum_{n=0}^{\infty} 1 /(2 n+1)^{2} \exp \left[-(2 n+1)^{2} \pi^{2} D t / 4 l^{2}\right]
$$

donde $m_{t}$ y $m_{\infty}$ se refieren a las masas de vapor de agua adsorbidas en un tiempo dado y en el tiempo teóricamente infinito respectivamente.

La serie de la ecuación (1) converge rápidamente, por ello se justifica acotarla hasta el segundo término ${ }^{(3,5)}$. Para obtener el coeficiente de difusión se resuelve la ecuación trascendente para el tiempo en el cual $m_{t} / m_{\infty}=1 / 2^{(3,7)}$. Empleando técnicas de aproximaciones sucesivas ${ }^{(8)}$ y teniendo en cuenta que la convergencia en la serie de aproximaciones sucesivas se garantiza desde la aproximación de primer orden entonces, despreciando el resto de las aproximaciones se obtiene que el coeficiente de difusión experimental del agua ambiental en la mezcla viene dado por la expresión ${ }^{(3)}$ :

$$
\mathrm{D}_{\exp }=0,196 l^{2} / t^{1 / 2}
$$

En un número importante de casos de interés práctico la difusión transcurre (para tiempos cortos) en un medio seminfinito $^{(3,5,9)}$, pues la primera reflexión del perfil de concentración 
para estos primeros tiempos aún no ha ocurrido. Si la difusión es independiente de la concentración la solución del segundo problema de contorno resulta:

$$
m_{t} / m_{\infty}=2\left(D t / \pi l^{2}\right)^{1 / 2}
$$

El comportamiento de $m_{t} / m_{\infty}$ en función de $\mathrm{t}^{1 / 2}$ para los instantes iniciales es lineal. A partir de la pendiente de esta función se puede determinar el coeficiente de difusión ${ }^{(3,5,7,9)}$ del soluto gaseoso en el material dado.

Los métodos antes descritos permiten el cálculo de los coeficientes efectivos de difusión. Sin embargo, ambos presentan limitaciones. En el primer caso es necesario usar procedimientos de interpolación para encontrar el tiempo medio, en tanto en el último procedimiento se emplean los primeros tiempos, donde precisamente los errores experimentales tienen un mayor peso.

Los métodos de computo actuales nos permiten, haciendo uso de los métodos de ajuste no lineales, programar el modelo difusivo representado por la ecuación (1), determinar los coeficientes de difusión y analizar con que certeza el modelo seleccionado explica o no el fenómeno de difusión del soluto en el material dado.

En el presente trabajo se describe el estudio de difusión de humedad en mezclas de moldeo empleadas en los procesos de fundición. Para ello, inicialmente se determinan los incrementos (decrementos) en masa húmeda de un grupo de mezclas autofraguantes usando técnicas gravimétricas clásicas ${ }^{7,9-}$ ${ }^{19)}$, análogas a las empleadas en el registro del incremento en peso de una muestra de material absorbente en interacción con un absorbato gaseoso para estudios de absorción y difusión en polímeros ${ }^{(7,10-12)}$, vidrios y dieléctricos ${ }^{(13,14)}$, zeolitas ${ }^{(15-}$ ${ }^{18)} \mathrm{y}$ sólidos inertes ${ }^{(19)}$ en general.

Mediante comparación de los incrementos porcentuales en masa húmeda de equilibrio para las diferentes mezclas estudiadas se comparan los poderes higroscópicos de las mismas y se analiza la influencia de sus diferentes constituyentes en la capacidad higroscópica de ellas.

Finalmente, haciendo uso de métodos de ajustes no lineales se determinan los coeficientes efectivos de difusión del agua atmosférica en los casos de los procesos absortivos de humedad a diferentes intemperies. Los resultados obtenidos están en el orden de lo descrito en la literatura, obteniéndose además una ligera dependencia de los coeficientes de difusión con la concentración como también se ha descrito.

\section{PARTE EXPERIMENTAL.}

\subsection{Características y propiedades fundamentales de los materiales utilizados.}

Las composiciones de algunas de las mezclas ensayadas se han mejorado aplicando técnicas de diseño de experimento $^{(20-22)}$.

En el caso de las mezclas con silicato de sodio (vidrio líquido) y proceso $\mathrm{CO}_{2}$ que emplean como desarenantes melaza, azúcar o mazut se diseñó un experimento factorial incompleto con relaciones de componentes y variable de operación $\left(1 / 33^{3}\right)^{(23)}$ en el cual las respuestas del sistema fueron la resistencia mecánica a la compresión a las 24 horas y la desmoldeabilidad. Tomando como criterio para la selección de la composición óptima para cada una de las mezclas ensayadas el balance adecuado entre estos parámetros se seleccionaron las
TABLA I. COMPOSICIÓN DE LAS MEZCLAS ESTUDIADAS.

\begin{tabular}{|c|c|c|c|c|c||}
\hline MEZCLAS & $\begin{array}{c}\text { ARENA } \\
\text { SÍLICE } \\
(\%)\end{array}$ & $\begin{array}{c}\text { SILICATO } \\
(\%)\end{array}$ & $\begin{array}{c}\text { TIEMPO DE } \\
\text { SOPLADO; } \\
\text { S }\end{array}$ & $\begin{array}{c}\text { ADITIVO } \\
\text { MELAZA } \\
(\%)\end{array}$ & $\begin{array}{c}\text { OTROS } \\
\text { COMPO- } \\
\text { NENTES (\%) }\end{array}$ \\
\hline $\mathrm{M}_{10}$ & 92,1 & 5,6 & 60 & 2,3 & - \\
\hline $\mathrm{G}_{1}$ & 90,29 & $4,85^{*}$ & 37 & - & $\begin{array}{c}\text { Esquistos } \\
\text { grafíticos } \\
\text { micáceos } \\
4,85\end{array}$ \\
\hline $\mathrm{A}_{3}$ & 95 & 3,9 & 40 & - & Azúcar 1,1 \\
\hline $\mathrm{E}_{1}$ & 94 & 3,63 & 7,5 & - & $\begin{array}{c}\text { Escoria 2,05 } \\
\text { Azúcar 0,32 }\end{array}$ \\
\hline $\mathrm{M}_{2}$ & 87 & 6,5 & - & 3,2 & $\begin{array}{c}\text { Sulfato de } \\
\text { sodio 3,3 }\end{array}$ \\
\hline $\mathrm{C}_{1}$ & 93,5 & $\begin{array}{c}5 \% \text { de } \\
\text { solución con }\end{array}$ & 5 & - & $\begin{array}{c}\text { Cemento 1,5 } \\
\text { azúcar }\end{array}$ \\
\hline $\mathrm{E}_{2}$ & 92,05 & 4,4 & - & - & $\begin{array}{c}\text { Pentoacetato } \\
\text { de glucosa } \\
3,55\end{array}$ \\
\hline $\mathrm{M}_{\mathrm{A} 5}$ & 94,2 & 4,9 & 60 & & Mazut \\
\hline $\mathrm{M}_{1}$ & 90,11 & $6,98^{*}$ & 41 & 2,9 & - \\
\hline
\end{tabular}

*En estos casos se empleó silicato de potasio y en el resto silicato de sodio, habitualmente empleado.

mezclas $\mathrm{M}_{10}, \mathrm{~A}_{3} \mathrm{y} \mathrm{M}_{\mathrm{A} 5}$ como las más apropiadas. Las composiciones del resto de las mezclas fueron encontradas empleando este mismo criterio y otros diseños factoriales.

De esta forma, las denominaciones $\mathrm{M}_{10^{\prime}} \mathrm{G}_{1}$, etc. se corresponden con la primera letra del componente que le ha dado nombre a la mezcla. El número que aparece como subíndice indica la composición de la mezcla que en el diseño dado ofrece las mejores propiedades o corresponde a una composición de propiedades adecuadas ubicada en la zona del simplex en estudio.

En la elaboración de las mezclas se empleó como material base arena sílice del tipo 1k 016 A (Norma Soviética GOST 2138-74.), procedente del yacimiento Casilda en las proximidades de Trinidad Sus características y composición química han sido descritas anteriormente ${ }^{(24)}$. Se trata de una arena de cuarzo con menos de un $2 \%$ de componente arcilloso y un contenido de $\mathrm{S}_{\mathrm{i}} \mathrm{O}_{2}$ entre el 96 y el $98 \%$. Durante el tamizado dejó más del $70 \%$ de sus granos en los tamices 02,016 y 01 con la particularidad de encontrarse un mayor residuo en el tamiz 02 que en el 01.

Se utilizó silicato de sodio de módulo 2,17 y densidad 1,49 $\mathrm{g} / \mathrm{cm}^{3}$; el cual cumple con las exigencias que se le plantean a este material en su condición de aglutinante. ${ }^{(25)}$

En dos de las mezclas estudiadas se empleó como aglutinante cemento Porland PP-250, en una de ellas en combinación con $\mathrm{CO}_{2}$.

Como endurecedor gaseoso se usó el $\mathrm{CO}_{2}$. La escoria de horno de cubilote y los esquistos grafíticos micáceos fueron utilizados como endurecedores sólidos, destacándose además que este último posee también propiedades desarenantes.

En la composición de la escoria ácida de horno de cubilote están presentes como máximo $40 \%$ de $\mathrm{SiO}, 27,57 \%$ de $\mathrm{CaO}$, $1,93 \%$ de $\mathrm{MgO}$ y $0,68 \%$ de $\mathrm{Fe}_{2} \mathrm{O}_{3}$ entre otros componentes.

Los esquistos grafíticos micáceo presentan entre un 4 y $5 \%$ de $\mathrm{C}, 0,6 \%$ de $\mathrm{S}, 16,79$ de $\mathrm{CaCO}_{3}$ y 88,44\% de cenizas. De ellos el 11,56\% constituyen sustancias volátiles. La composición mineralógica presenta: $70 \%$ de mica moscovita con impregnación de materia carbonosa, 19\% de material carbo- 
noso, $1 \%$ de mica biolítica y 10\% de cuarzo. La fracción usada fue de $0,088 \mathrm{~mm}$.

En la mezcla $\mathrm{M}_{2}$ se empleó como endurecedor con propiedades desarenantes el sulfato de sodio, sólido cristalino de color blanco, de olor característico y muy higroscópico. Se caracteriza por una pureza expresada como sulfato de sodio entre 93 y 95\% y acidez libre expresada como ácido sulfúrico al $1,5 \%$ con su contenido de cloruro de sodio $2,5 \%$ y un 0,8 de sustancias insolubles.

Entre los aditivos desarenantes usados se encuentran la melaza, el azúcar de barrido y el serrín. La melaza empleada, subproducto de la industria azucarera, se ajusta a las especificaciones para su uso en la fundición según la norma cubana NC-8105-81(Cantidad mínima de sólidos solubles en la miel a $36 \%$, cantidad mínima de azúcares totales $\simeq 52 \%$ y densidad $1,3 \mathrm{~g} / \mathrm{cm}^{3}$ ) y cumple con las reglamentaciones para su almacenamiento y conservación con posterioridad a la entrega según la norma cubana NC-8121-85.

\subsection{Elaboración de las mezclas y probetas.}

Las mezclas se confeccionaron en base a $2 \mathrm{Kg}$. de masa total para cada composición. Después de pesados cada uno de los componentes en una balanza de exactitud 0,1g, se mezclaron los componentes sólidos durante 2 minutos, luego se añadió la emulsión preparada con los componentes líquidos premezclados (tipo aglutinante complejo) ${ }^{(26)}$ y finalmente se mezclaron los componentes sólidos y líquidos.

Posteriormente se confeccionaron las probetas normalizadas $(50 \times 50 \mathrm{~mm})$ compactadas a tres golpes; las que fueron gaseadas con $\mathrm{CO}_{2}$ en los casos requeridos a presión de 0.2 MPa. (2 atm.) durante el tiempo establecido. [Tabla I.] Finalmente, a partir de estas probetas se elaboraron las pastillas finitas de espesor $l$ y diámetro $d(d<<l)$, permeables por una cara.

\subsection{Ensayo de humectación.}

Las pastillas elaboradas se sometieron al ensayo de humectación en una cámara climatizada, especialmente diseñada para estos fines ${ }^{(3)}$, a humedades relativas $(\mathrm{Hr}$.) y temperaturas constantes. Las características de esta cámara han sido descritas con anterioridad ${ }^{(27)}$. Los incrementos (decrementos) relativos en masa húmeda se determinaron en una balanza digital de exactitud $0.1 \mathrm{mg}$.

Las ganancias relativas en masa húmeda en el tiempo para cada mezcla y humedad relativa del medio exterior se determinaron por la expresión ${ }^{(3,28)}$ :

$$
\bar{C}(t)=\left\{\left[m(t)-m\left(t_{0}\right)\right] / m\left(t_{0}\right)\right\} * 100 \%
$$

donde $m(t)$ es la masa después de la humectación (la masa del producto húmedo en el tiempo $t$ de exposición a la atmósfera dada), la que se mide a intervalos de tiempos preferiblemente fijos; y $m\left(t_{0}\right)$ es la masa de la muestra en el instante inicial del ensayo (masa del producto "seco").

En la práctica la masa en el equilibrio, teóricamente en el infinito $\left(m_{\infty}\right)$, se toma como aquel valor de la masa de vapor de agua absorbida o desorbida que se repite varias veces en el experimento o cuyo incremento o decremento está en el orden de exactitud de la balanza.

Para el cálculo de los coeficientes efectivos de difusión se resolvió la serie de la ecuación (2), aproximándola hasta el segundo término. El modelo difusivo así obtenido se programó, para posteriormente realizar el ajuste de las datas experimentales empleando técnicas no lineales. El número de iteraciones ejecutadas en cada caso lo determinó la convergencia del valor del parámetro difusivo estimado en cada iteración con el calculado en la iteración precedente.

\section{PRESENTACIÓN Y DISCUSIÓN DE LOS RESULTADOS}

En las figuras 1-9 aparecen los gráficos de $\bar{C}$ Vs $t$ para las mezclas estudiadas, en ellas se pueden apreciar las curvas de absorción y desorción del agua atmosférica, en rangos de humedades relativas ambientales típicas del clima tropical cubano (60 - 95\% Hr.). La mayoría de ellas manifiestan una única concavidad hacia el eje temporal, sin la existencia de puntos de inflexión en los tiempos ensayados. Además se puede apreciar que cuando $t \rightarrow 0$ las pendientes de las curvas $t \bar{C} / d t \rightarrow \pm \infty$. Lo antes dicho justifica ${ }^{(3)}$ que tanto la absorción de vapor de agua a altas humedades relativas, como la desorción para las bajas Hr. en estos casos constituyen procesos puramente difusivos.

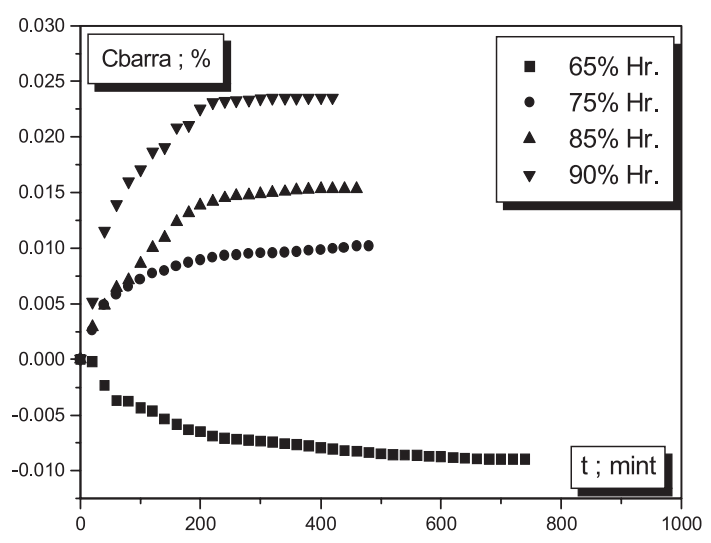

Fig.1. Curvas de la cinética de ganancia o pérdida en masa húmeda para la mezcla M10.

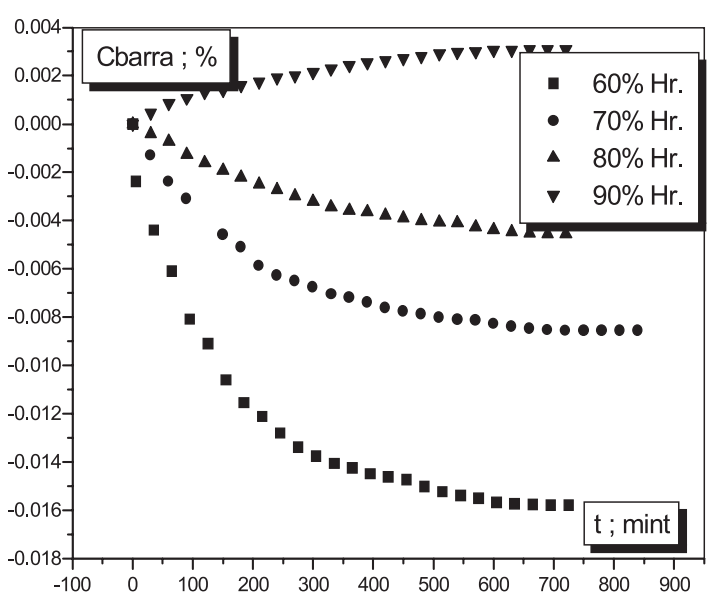

Fig. 2. Curvas de la cinética de ganancia o pérdida en masa húmeda para la mezcla G1. 


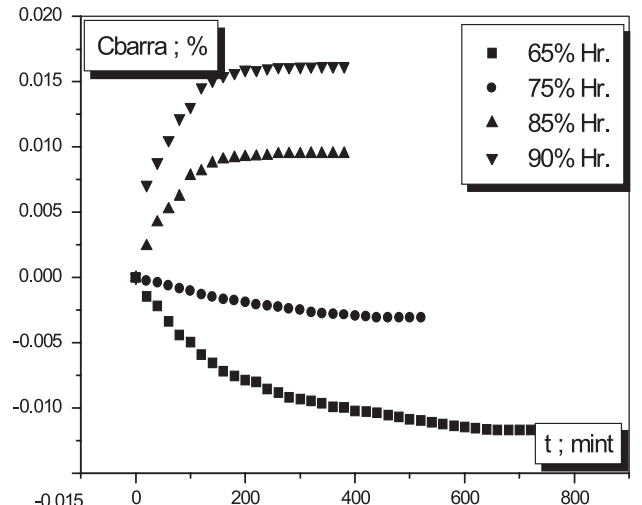

Fig. 3.Curvas de la cinética de ganáncia o pérdida en masa húmeda para la mezcla A3.

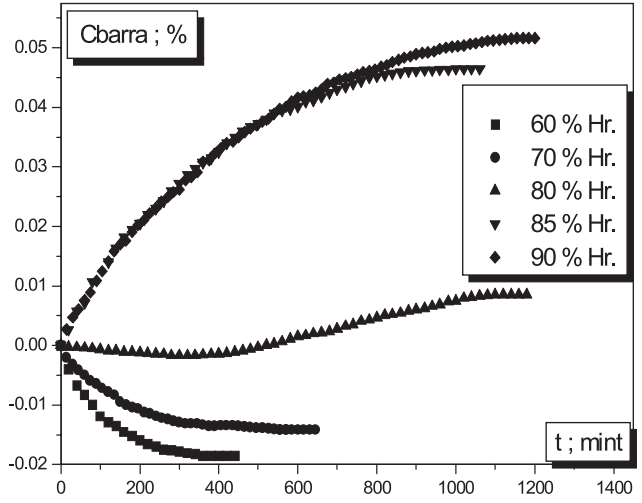

Fig. 5.Curvas de la cinética de ganancia o pérdida en masa húmeda para la mezcla M2.

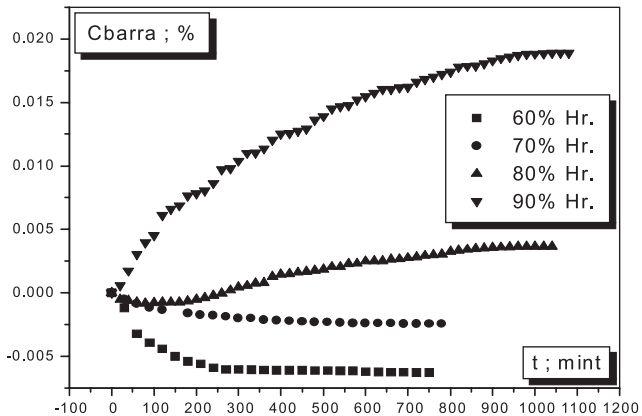

Fig. 7. Curvas de la cinética de ganancia o pérdida en masa húmeda para la mezcla E2.

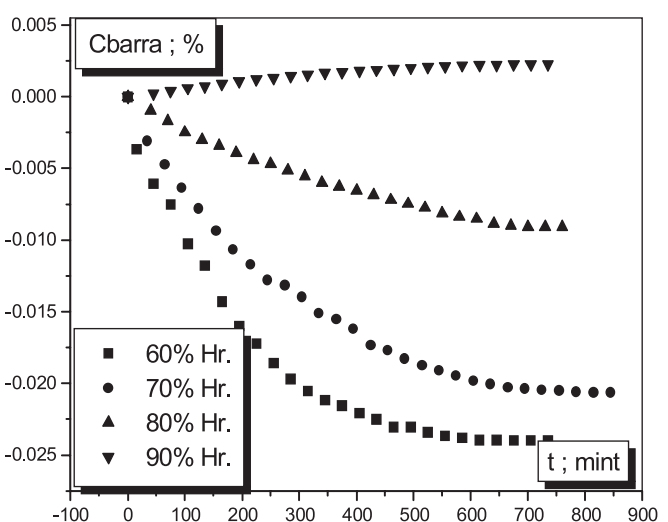

Fig. 9. Curvas de la cinética de ganancia o pérdida en masa húmeda para la mezcla M1.

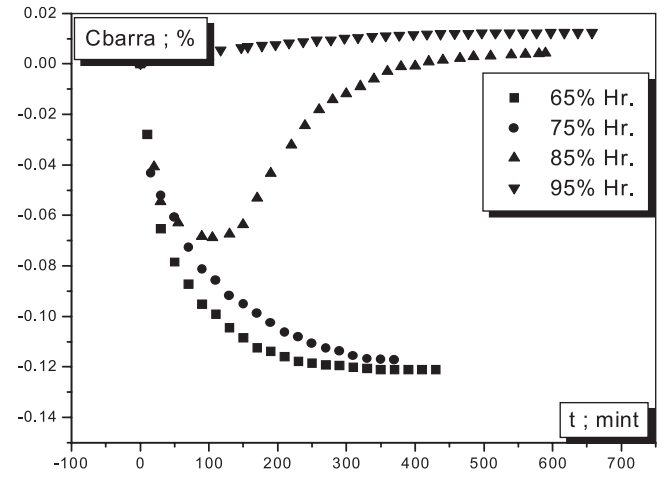

Fig. 4. Curvas de la cinética de ganancia o pérdida en masa húmeda para la mezcla E1.

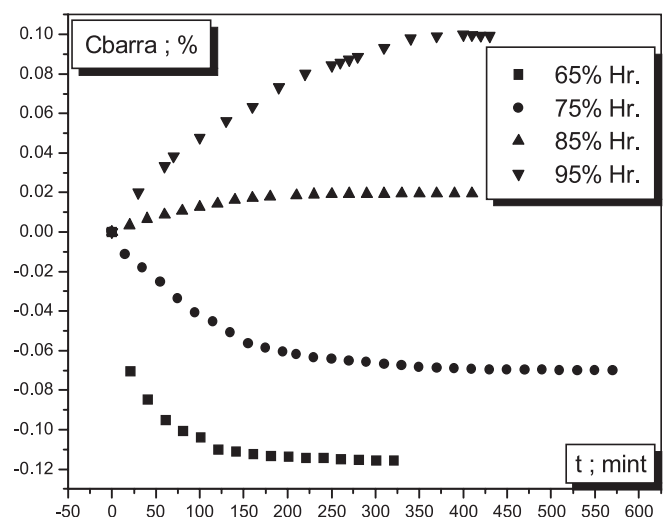

Fig. 6. Curvas de la cinética de ganancia o pérdida en masa húmeda para la mezcla $\mathrm{C} 1$.

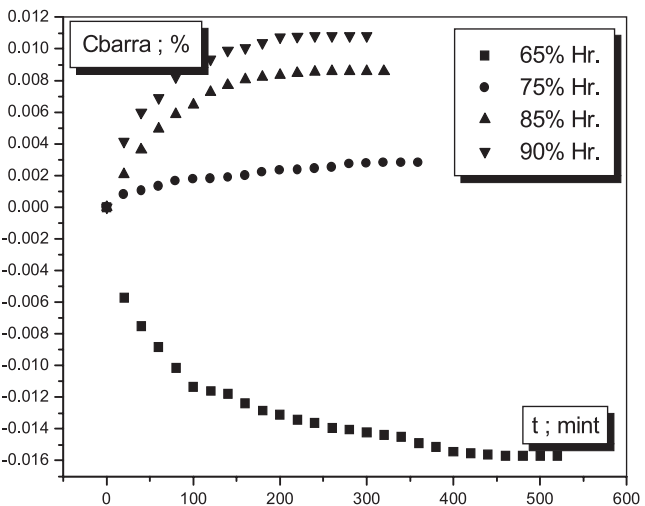

Fig. 8. Curvas de la cinética de ganancia o pérdida en masa húmeda para la mezcla Ma5.

Constituyen una excepción del comportamiento antes descrito las curvas de absorción-desorción para humedades relativas en el entorno del $80 \%$ para las mezclas $\mathrm{E}_{1}, \mathrm{E}_{2} \mathrm{y}_{2}$. Esto se identifica por la presencia de un punto de inflexión en las curvas de $\bar{C}$ Vs $t$. El proceso se inicializa con un decremento de la humedad porcentual en la mezcla, de manera que al inicio la deshidratación es gobernada por la difusión, pues $d \bar{C} / d t \rightarrow-\infty \quad$ cuando $t \rightarrow 0$. Después, al cabo de un tiempo prudencial, se produce un cambio en la concavidad de la curva y un incremento posterior de la humedad en la mezcla tiene lugar. De esta forma, el fenómeno de adsorción - desorción, a esta intemperie, se caracteriza por la presencia de un punto de inflexión en la curva que describe su cinética y el propio proceso de pérdida difusiva de masa húmeda crea las 
condiciones necesarias para que tenga lugar la nucleación de una nueva fase ${ }^{(3,29)}$.

En el caso de la mezcla $\mathrm{E}_{1}$ esto pudiera deberse a un alto contenido de humedad inicial en la mezcla con respecto a la humedad ambiental, que combinado con el bajo tiempo de soplado con $\mathrm{CO}_{2}$ retarda o no favorece el proceso de formación del gel de ácido silícico. Entonces, la deshidratación inicial de la mezcla, en presencia del gas carbónico ambiental favorece la formación de la placa vidriosa de silicato de sodio, altamente porosa y capilar, responsable del proceso absortivo ulterior. Para las mezclas $\mathrm{E}_{2}$ y $\mathrm{M}_{2}$ ocurre algo parecido; en estos casos, con la diferencia de que el soplado con gas carbónico está ausente y la formación del gel ocurre solo a cuenta del $\mathrm{CO}_{2}$ atmosférico. De esta forma, el propio proceso de pérdida difusiva de masa húmeda crea las condiciones necesarias para que tenga lugar la nucleación de una nueva fase ${ }^{(3,29)}$.

La mayoría de las mezclas sometidas al ensayo de higroscopicidad decrecen su masa húmeda en el rango de Hr. que se encuentran por debajo del $80 \%$. Ello se debe a que la presión parcial del vapor de agua dentro del sólido (dado por el contenido inicial de humedad), es superior a las correspondientes presiones parciales de las atmósferas exteriores. Esto hace posible la existencia de un gradiente de concentraciones de humedad y por tanto el inicio de un flujo difusivo del agua hacia el medio que rodea al sólido, hasta tanto se igualen las presiones dentro y fuera de la mezcla.

Tabla II. VAlores de $\overline{\mathrm{C}}_{\infty}$ a Diferentes Hr. para las distintas Mezclas ESTUDIADAS.

\begin{tabular}{|c|c|c|c|c|c|c|c|}
\hline \multirow{2}{*}{ MEZCLAS } & \multicolumn{7}{|c||}{ VALORES DE C A DIFERENTES Hr. (\%) } \\
\cline { 2 - 8 } & 60 & 65 & 70 & 75 & 80 & 85 & 90 \\
\hline$M_{10}$ & & $-0,0089$ & & 0,0102 & & 0,0153 & 0,0235 \\
\hline$G_{1}$ & $-0,0158$ & & $-0,0085$ & & $-0,0045$ & & 0,0031 \\
\hline$A_{3}$ & & $-0,0117$ & & $-0,0031$ & & 0,0095 & 0,0162 \\
\hline$E_{1}$ & & $-0,1211$ & & $-0,1172$ & & 0.0042 & 0,0123 \\
\hline$M_{2}$ & $-0,0186$ & & $-0,0142$ & & 0,0085 & 0,0464 & 0,0516 \\
\hline$C_{1}$ & & $-0,1156$ & & $-0,0700$ & & 0,0195 & 0,0991 \\
\hline$E_{2}$ & $-0,0063$ & & $-0,0025$ & & 0,0036 & & 0,0189 \\
\hline$M_{A 5}$ & $-0,0157$ & & 0,0028 & & 0,0086 & 0,0108 & \\
\hline$M_{1}$ & $-0,0240$ & & $-0,0207$ & & $-0,0091$ & & 0,0022 \\
\hline
\end{tabular}

La Tabla II. refiere los valores de la concentración de equilibrio para las diferentes intemperies $\left(\bar{C}_{\infty}\right)$, es decir los valores de $\bar{C}$ para los cuales las pastillas no absorben ni desorben agua atmosférica. Se puede apreciar la alta capacidad higroscópica de los desarenantes tipo azucarados en comparación con el resto de los desarenantes en todo el rango de humedades relativas ensayados. Particularmente significativo resulta el caso de la mezcla $M_{2}$ en la cual además está presente el sulfato de sodio. Resaltan también las buenas propiedades higroscópicas del desarenante grafito frente a los del tipo azucarado y del aglutinante silicato de potasio comparado con el silicato de sodio. Resulta paradójico las buenas propiedades higroscópicas de la mezcla $M_{1}$ que contiene melaza, desarenante de alto poder higroscópico como se dijo antes, frente a otras mezclas con silicato de sodio y melaza. Esto último sugiere que el alto poder higroscópico de la mezcla se acrecienta cuando se combina la melaza con el silicato de sodio.

El alto poder higroscópico que manifiesta de la mezcla $\mathrm{C}_{1}$, a altas humedades, pudiera ser explicado por la necesidad de agua para el fraguado del cemento.
TABLA III. COEFICIENTES EFECTIVOS DE DIFUSIÓN EN LAS MEZCLAS ESTUDIADAS.

\begin{tabular}{||c|c|c|c||}
\hline \multirow{2}{*}{ MEZCLAS } & $\mathrm{Hr} .(\%)$ & $l ; \mathrm{mm}$ & $\begin{array}{c}\mathrm{D}_{\text {EXP. } 10^{-4}} \\
\mathrm{~mm}^{2} / \mathrm{s}\end{array}$ \\
\hline \multirow{3}{*}{$\mathrm{M}_{10}$} & 75 & 2,70 & 4,70 \\
\cline { 2 - 4 } & 85 & 2,70 & 4,36 \\
\cline { 2 - 4 } & 90 & 2,16 & 3,62 \\
\hline $\mathrm{G}_{1}$ & 90 & 6,00 & 8,83 \\
\hline \multirow{2}{*}{$\mathrm{A}_{3}$} & 85 & 3,51 & 10,68 \\
\cline { 2 - 4 } & 90 & 1,78 & 3,36 \\
\hline $\mathrm{E}_{1}$ & 95 & 2,45 & 1,85 \\
\hline \multirow{2}{*}{$\mathrm{M}_{2}$} & 85 & 3,70 & 3,47 \\
\cline { 2 - 4 } & 90 & 3,75 & 2,07 \\
\hline \multirow{2}{*}{$\mathrm{C}_{1}$} & 85 & 2,35 & 3,51 \\
\cline { 2 - 4 } & 95 & 2,30 & 2,21 \\
\hline $\mathrm{E}_{2}$ & 90 & 6,80 & 7,21 \\
\hline \multirow{2}{*}{$\mathrm{M}_{\mathrm{A} 5}$} & 75 & 3,31 & 8,67 \\
\cline { 2 - 4 } & 85 & 3,21 & 8,58 \\
\hline $\mathrm{M}_{1}$ & 90 & 2,82 & 8,20 \\
\hline
\end{tabular}

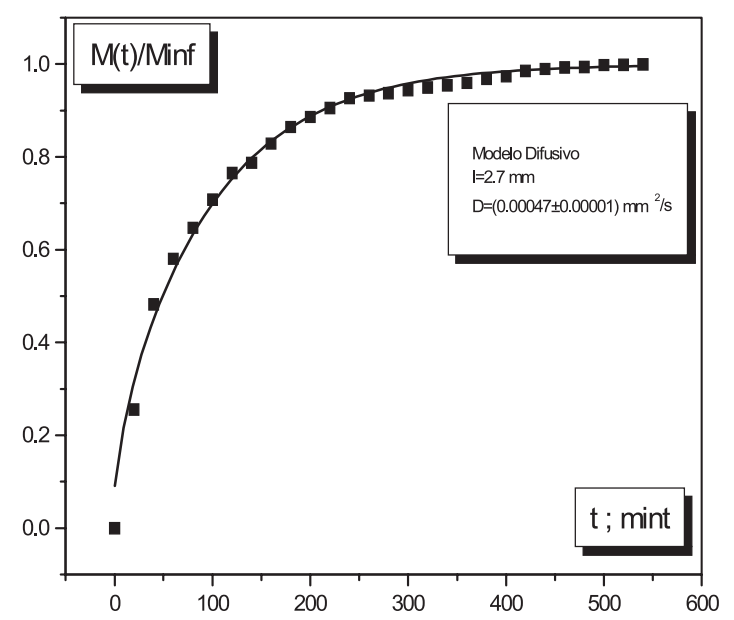

Fig. 10. Ajuste de la data experimental según el modelo difusivo para la mezcla M 10 a una intemperie de $75 \% \mathrm{Hr}$.

Los coeficientes efectivos de difusión para el caso de los proceso absortivos puros y calculados empleando técnicas de ajuste no lineal manifiestan una suave dependencia con la concentración de equilibrio(Tabla III.), pues aunque todos están en un mismo orden, se puede comprobar la tendencia de éstos al decrecimiento con el incremento de la concentración de humedad en la mezcla. En la Fig. 10 se ha visualizado, a modo de ejemplo, el procedimiento empleado para el ajuste del parámetro difusivo.

Se puede inferir que en los primeros momentos de la experiencia difusiva, como $\bar{C}(\mathrm{t})$ es muy pequeño, los coeficientes efectivos de difusión en la mezcla ensayada serán muy elevados, siendo mayor la tendencia a la variación para los pequeños $\bar{C}(\mathrm{t})$, por lo que una rápida movilidad de los perfiles de concentraciones se tendrán en los mismos, lo que hace que el experimento difusivo no se pueda considerar, en los tiempos de interés, como un problema de transporte en un medio sem-infinito, ya que la primera reflexión del perfil C ( $\mathrm{x}$, t) se manifiesta, en las pastillas permeables por una cara y de espesores pequeños en los instantes iniciales de la experien- 
cia, en tanto para tiempos prolongados del ensayo se está en presencia de múltiples reflexiones del perfil de concentraciones, lo cual justifica la elección del modelo de pastilla finita de espesor $l$ en las muestras estudiadas cuando los ensayos de higroscopicidad son prolongados.

\section{CONCLUSIONES}

La cinética de ganancia o pérdida relativa de humedad es gobernada en las mezclas de moldeo estudiadas por la difusión física del agua atmosférica hacia o desde la mezcla, excepto en el entorno del $80 \%$ Hr. para las mezclas $\mathrm{E}_{1}, \mathrm{E}_{2} \mathrm{y} \mathrm{M}_{2}$.

El ensayo de higroscopicidad, haciendo uso de la pastilla permeable por una cara y el modelo teórico de pastilla finita, permite la obtención de los coeficientes efectivos de difusión, en el caso de procesos absortivos de agua higroscópica, independientemente de la geometría que en la práctica adopte la mezcla de moldeo, por lo cual la metodología explicada puede ser usada con los fines aquí descritos.

Se comprueba el efecto que tiene la concentración de agua incorporada a las mezclas en sus parámetros difusivos. En la medida en que crecen los contenidos de agua en las mezclas, los coeficientes efectivos de difusión decrecen.

Las mezclas $G_{1}, E_{1}$ y $E_{2}$ manifiestan las mejores propiedades higroscópicas, en tanto las mezclas que usan aditivos azucarados, fundamentalmente las que emplean melaza, poseen un alto poder higroscópico. Por ello, la necesidad de establecer normativas que permitan reducir al máximo los contenidos de agua atmosférica que se adquiere por los machos durante su permanencia a determinadas intemperies con lo cual disminuirían las probabilidades de surgimiento de los defectos asociados con la absorción de humedad por las mezclas y con ello los índices de rechazo lo que posibilitará producciones más eficientes y económicas.

\section{BIBLIOGRAFÍA}

1. A. Sanders Clyde. Foundry 99(10) (1971) 110.

2. New Tecnology at the Casting Congress. $75^{\text {th }}$ AFS Casting Congress Foundry 99(5) (1971) 72.

3. E. Valencia. Ph.D. Disertación. Higroscopicidad en mezclas de arena y melaza para la fundición cubana. pp.47 - 80. Higroscopicidad en los revestimientos de los electrodos básicos y mezclas de moldeo para la fundición cubana. UCLV. Sta. Clara.(Cuba).1992.

4. C.M. Salcines Merino. Higroscopicidad. pp.383 -284 Tecnología de fundición. Tomo I. Editorial Pueblo y Educación. Habana.(Cuba).1985.

5. J. Crank. Diffusion in a plane sheet. pp. 41-68. The Mathematics of Diffusion. $2^{\text {nd }}$ ed. Clarendon Press. Oxford. 1975.

6. W. Jost. Diffusion in solids, liquids, and gases. Academic Press. New York. 1960.
7. P. Neogi. Transport phenomenon in polymer membranes. pp.173-209. Diffusion in Polymers. Edited by P. Neogi. Marcel Dekker, inc. New York Basel - Hong Kong.1996.

8. L. Elsgolttz. Principios de las transformaciones de contracción. pp. 51 - 56. Ecuaciones diferenciales y cálculo variacional. Edit. Mir. Moscú 1983.

9. K- I. Okamoto. Sorption and diffusion of water vapor in polimide films. pp. $265-278$ Polymides. Fundamentals and applications. Edited by K. G. Malay \& K.L. Mittal. Marcel Dekker, inc. New York - Basel - Hong Kong.1995.

10. B. Vázquez, and J. San Roman. Polymeric hydrophilic hydroxyls with flexible hydrophobic chains- control of the hydration and interactions with water molecules. Macromolecules. 30(26) 8440 - 8446 (1997).

11. C. Peniche, D. Zaldivar, A. Gallardo, \& J. San Román. J. Appl. Polym. Sci. 54(1994) 959.

12. C. Peniche. \& W. Argüelles. Polym. Intern. 38(1995) 45.

13. M. Tomosawa. H. Li. and K.M. Davis. Water diffusion, oxygen vacancy annihilation and structural relaxation in silica glasses. J. Non-Cryst. Solids. 179(1994) 162-169.

14. B.M. Taréiev. Propiedades con respecto a la humedad de los dieléctricos. pp.383 - 400 Física de los materiales dieléctricos. Editorial MIR. Moscú.(URSS). 1978.

15. L. Abrams and A.J. Owens. : A descriptive equation for diffusion / sorption date. J. Phys - Chem. 90(1986) $2300-2302$.

16. M.W. Ackley and R.T. Yang. Diffusion in Ion - Exchanged Clinoptilolites. A.I. Ch.E. Journal 371645 -1656 (1991).

17. M.W. Ackley, R.F. Giese and R.T. Yang. Zeolite 12(1992) 770 - 788.

18. D.M. Ruthven. Principy of the Adsorption and Process of Adsorption. A Wiley - Interscience Publication. John Wiley and Sons. 1984.

19. J.K. Baird and J-S. Chen. Theory of time - lag diffusion method for the case of outgassing solid. J. Mater Res. 6(1993) 1455 - 1461.

20. S. Akhnasarova and V. Kafarov. Mixture Design. pp. 240 - 295. Experiment optimization in chemistry and chemical engineering. MIR Publishers. Moscow(URSS). 1982.

21. J.A. Cornell and J.W. Gorman. Factorial designs plants for process variables in mixture experiments. J. Quality Technology 16(1) 1984.

22. O.O. Kenworty. Factorial experiment with mixtures using ratios. Industrial Quality Control 19(12) $24-26$ (1963).

23. J.E. Hernández Ruiz y otros. Diseño factorial incompleto con relaciones y variables de proceso y la cinética de absorción - desorción del agua atmosférica en mezclas de moldeo con vidrio líquido y proceso $\mathrm{CO}_{2}$. Construcción de Maquinarias 20(1) 43 - 51 (1995).

24. C.M. Salcines Merino. Arenas y arcillas de moldeo. pp. 52 -57.Tecnología de fundición. Tomo I. Editorial Pueblo y Educación. Habana.(Cuba).1985.

25. The Distillers Company (Carbon Dioxide) Ltd. El proceso silicato $\mathrm{CO}_{2}$. Estado actual. Colada 13(11) 257 - 259. (1980).

26. D. Warren, Perfeccionamiento del proceso de elaboración de mezclas con vidrio líquido en Inglaterra. British Foundry - men. 64(12) 1971.

27. J.E. Hernández, E. Valencia Morales y E. Villar Cociña, Influencia de la humedad en el comportamiento de la resistencia a la compresión en mezclas de moldeo. Boletín de la Sociedad Española de Cerámica y Vidrio 40(2) 107111 (2001)

28. E. Valencia. Hygroscopic Behavior of Certain Basic - Coated Electrodes in Wet Tropical Climate. Welding International. 8(3) 1994 Rev. Soldadura. Madrid. 23(2) 94 - 103 (1993).

29. I.V. Ryzhkov y V.S. Tolstoy, Los Principios Físico-Químico de la Formación de las Propiedades de las Mezclas con Vidrio Líquido, en Ruso, editado por N.N. Sorokin(Editorial Visha Shkola, Jarkov, 1975).

Recibido: 24.11 .00

Aceptado: 14.01 .02 